\title{
Effects of increased matrix metalloproteinase-9 expression on skeletal muscle fibrosis in prolonged alcoholic myopathies of rats
}

\author{
JIANFENG WANG $^{1}$, YANLI LIU ${ }^{1}$, LI ZHANG $^{2}$, JUN JI$^{2}$, BING WANG $^{3}$, \\ WEI JIN ${ }^{4}$, CHENGHONG ZHANG ${ }^{3}$ and HAIYING CHU ${ }^{4}$ \\ ${ }^{1}$ Department of Neurology; ${ }^{2}$ Central Laboratory, Dalian Central Hospital, Dalian, Liaoning 116033; ${ }^{3}$ Morphology \\ Laboratory; ${ }^{4}$ Department of Histology and Embryology, Dalian Medical University, Dalian, Liaoning 116044, P.R. China
}

Received June 22, 2011; Accepted September 5, 2011

DOI: $10.3892 / \mathrm{mmr} .2011 .592$

\begin{abstract}
This study evaluated the effects of matrix metalloproteinase-2 (MMP-2) and matrix metalloproteinase-9 (MMP-9) on injured gastrocnemius, soleus and plantaris muscles, induced by alcohol in rats. A total of 60 male SpragueDawley rats $(2.5$ months old, $200 \pm 20 \mathrm{~g})$ were divided into 6 groups: i) untreated skeletal muscle and analyzed 2 weeks later (A1 group, 5 rats); ii) untreated skeletal muscle and analyzed 6 weeks later (A2 group, 5 rats); iii) untreated skeletal muscle and analyzed 12 weeks later (A3 group, 5 rats); iv) injured skeletal muscle and analyzed 2 weeks later (B1 group, 15 rats); v) injured skeletal muscle and analyzed 6 weeks later (B2 group, 15 rats); and vi) injured skeletal muscle and analyzed 12 weeks later (B3 group, 15 rats). The injured and uninjured muscles were observed by light microscopy and polarization microscopy. The MMP activity was evaluated through zymography, and messenger RNA (mRNA) of MMP-9 and of MMP-2 were assessed by RT-PCR. The expression of MMP-9 was assessed by Western blot analysis. The plantaris and gastrocnemius muscles in the rats subjected to alcohol ingestion were found to have a high expression of MMP-9, but not of MMP-2. Picrosirius red staining was used to assess whether increased fibrosis in the skeletal muscle was associated with alcohol exposure in rats. The study indicated that alcohol may be involved in the skeletal muscle interstitial fibrosis in our model of alcohol-exposed rats through increased MMP-9 expression, and that this increased expression may aggravate the development of prolonged alcohol muscle injury.
\end{abstract}

\section{Introduction}

Prolonged alcoholic myopathy affects up to two thirds of all alcohol misusers and is characterized by selective atrophy of

Correspondence to: Professor Haiying Chu or Dr Jianfeng Wang, Department of Histology and Embryology, Dalian Medical University, Dalian, Liaoning 116044, P.R China

E-mail: haiying07@yahoo.com.cn; jfwang06@yahoo.com.cn

Key words: prolonged alcoholic myopathy, matrix metalloproteinase-9, skeletal muscle fibrosis type II (glycolytic, fast-twitch, anaerobic) fibers. Common features observed in chronic alcoholics include weakness and difficulties in gait, with objective symptoms of reduced skeletal muscle strength and loss of total skeletal muscle mass. These symptoms have previously been described as neuropathological in origin. However, biochemical lesions and/or the presence of a defined myopathy may occur in alcoholics as a direct consequence of alcohol misuse. The myopathy occurs independently of peripheral neuropathy, malnutrition and overt liver disease and is more common than other alcohol-induced diseases, such as cirrhosis (15-20\% of chronic alcoholics), peripheral neuropathy (15-20\%), intestinal disease (30-50\%) or cardiomyopathy (15-35\%) (1). Although musculoskeletal symptoms are common in alcoholics, little is known of the prevalence of muscle pathology in this group.

Matrix metalloproteinases (MMPs) are a family of zinc-dependent endopeptidases that are associated with the breakdown of constituents of the extracellular matrix (ECM) and this is tightly regulated by endogenous tissue inhibitors of MMPs (TIMPs) (2). Distinct expression patterns of MMPs may reflect various stages of myopathy. Inflammatory myopathies such as polymyositis and sporadic inclusion body myositis show a strong upregulation of MMP-9 and, to a lesser extent, of MMP-2 at atrophic and inflamed myofibers $(3,4)$. Amyotrophic lateral sclerosis-affected biopsies show MMP-9 immunoreactivity and, to a lesser extent, MMP-2 and MMP-7 at normal-sized and atrophic myofibers. Biopsies of spinal muscle atrophy show MMP-9 and MMP-7 immunoreactivity at normal sized and atrophic myofibers, while MMP-2 immunoreactivity was undetectable. In chronic axonal neuropathies, MMP-9 immunoreactivity is observed at normal-sized and atrophic myofibers (5).

In addition, MMP-9 is elevated in the serum of alcohol abusers (6). One of the most striking metabolic abnormalities produced by chronic alcohol abuse is the gradual reduction in muscle mass, which appears to be associated with the atrophy of type II (glycolytic, fast-twitch, anaerobic) fibers (7). The knowledge of the possible roles of MMPs in muscle biology and pathology is currently limited. Due to the probable role of distinct MMPs in myopathies, we attempted to investigate the effects of MMP-2 and MMP-9, on muscle atrophy and myofibrosis during prolonged alcoholic myopathy using a rat model of alcohol use. 


\section{Materials and methods}

Animal treatment. All experiments were performed with specific pathogen-free, male Sprague-Dawley rats (2.5 months old, 180-200 g; Dalian Medical University Experimental Animal Center, Dalian, LiaoNing, China). Rats were housed in a controlled environment, in an air-conditioned $\left(20-25^{\circ} \mathrm{C}\right)$, humidity-controlled (40-60\%) animal house with a $12 \mathrm{~h}$ light-dark cycle, commencing at 8:00 am, and had access to water and rat chow ad libitum for at least one week prior to the start of the study. A total of 60 male Sprague-Dawley rats were randomly divided into a control group (group A, 15 rats) or an experimental group (group B, 45 rats). Group A was then randomly divided into 3 subset groups (A1-A3). Each group consisted of 5 rats. Group B was randomly divided into 3 subset groups (B1-B3). Each group consisted of 15 rats. All rats were treated intragastrically by gavage. Treated rats (experimental groups) were fed a modified, standard, nutritionally-complete diet containing $35 \%$ of total calories from ethanol $(7,8)$. These rats were orally gavaged with $4-10 \mathrm{~g} / \mathrm{kg} /$ day body weight $(\mathrm{BW})$ of ethanol, in increasing doses. Untreated rats (control groups) were fed an identical diet in which the ethanol had been replaced by an isocaloric amount of glucose (9). A modified, standard, nutritionally-complete diet is as follows: an egg and $85 \mathrm{ml}$ of corn oil were added to a standard, nutritionally-complete diet, and then cooked. Some fish liver oil and multi-vitamins were added to the cooked diet and mixed. In the diet, the protein vs. fat vs. carbohydrate (energy) ratio was 17 vs. 31 vs. $52 \%$, the same as the energy ratio of the components in Lieber-Decarli liquid feeds. The diets were prepared daily. The A1 and B1 groups were analyzed 2 weeks later, the A2 and B2 groups were analyzed 6 weeks later and the A3 and B3 groups were analyzed 12 weeks later. At 2, 6 and 12 weeks, these rats were euthanized by an overdose of anesthetic, and skeletal muscles, including the gastrocnemius, soleus and plantaris from the posterior limb, were rapidly dissected.

Histochemistry assay. Rats were anesthetized (and subsequently euthanized) by an intraperitoneal injection of ketamine and xylazine. The triceps surae muscle group (consisting of the soleus, plantaris and gastrocnemius muscles) was dissected free of the surrounding tissues and snap-frozen in liquid nitrogen. Subsequently, the frozen muscle samples were serially sectioned at $-20^{\circ} \mathrm{C}$ with a cryostat. We performed a battery of histochemical stainings on the serial sections, including hematoxylin and eosin (H\&E) and $\beta$-nicotinamide adenine dinucleotide-tetrazolium reductase (NADH-TR) staining.

Assessment of skeletal muscle fibrosis. Collagen was detected under polarized light on muscle sections prepared by the picrosirius red technique, performed as previously described (10). Briefly, sections, deparaffinized with xylenes, were rehydrated in a descending series of ethanols in distilled water. The hydrated sections were immersed in $0.2 \%$ aqueous phosphomolybdic acid for $2 \mathrm{~min}$, rinsed in distilled water and were then stained for 60 min with $0.1 \%$ Sirius Red F3BA (Pfal and Bauer; Stamford, CT, USA) in saturated aqueous picric acid, $\mathrm{pH}$ 2.0. The sections were washed for $2 \mathrm{~min}$ in $0.01 \mathrm{~N} \mathrm{HCl}$, rinsed for $50 \mathrm{sec}$ in $70 \%$ ethanol, dehydrated in 3 changes of absolute alcohol, cleared in xylene and mounted in a non-aqueous mounting medium. Picrosirius red-stained sections were detected by polarization microscopy, under which collagen type I fibers appear yellow or red and collagen type III fibers appeared green. Picrosirius red-stained sections at X100 magnification were projected onto a computer screen and levels of interstitial fibrosis were assessed using the microscope computer Image-Pro 4.5 software (Media Cybernetics, Silver Spring, MD, USA). Tissues in which collagen occupied more than $5 \%$ of the total tissue area were scored as collagen-positive. Five views from collagen-positive fields were randomly selected for analysis. Total areas of collagenpositive fields were detected and then the medial absorbance (MA) was determined.

$M M P-2 / M M P-9 R T-P C R$. Total RNA was extracted from frozen gastrocnemius, soleus and plantaris muscle samples from rats exposed to alcohol at 2, 6 and 10 weeks, respectively, with TRIzol Reagent (Invitrogen Life Technologies, Carlsbad, CA, USA). Frozen muscle samples were mechanically homogenized on ice in $1 \mathrm{ml}$ of ice-cold TRIzol reagent. Total RNA was solubilized in RNase-free water and quantified by measuring the optical density (OD) at $260 \mathrm{~nm}$. RNA purity was ensured by obtaining a 260/280 $\mathrm{nm}$ OD ratio $>1.70$. The PCR amplifications were carried out using the PrimeScript RT-PCR kit (Takara, Japan). A total of $25 \mu \mathrm{l}$ of polymerase chain reaction (PCR) solution contained $1.5 \mathrm{mM} \mathrm{MgCl}_{2}, 0.2 \mathrm{mM}$ of all deoxynucleotide triphosphates, $0.4 \mathrm{mM}$ of each forward and reverse primer and 0.1 units of Taq DNA polymerase (Gibco). The complementary DNAs (cDNAs) were used to amplify a 421 bp fragment using specific primers for the MMP-2 by PCR, a 496 bp fragment using specific primers for the MMP-9 and a $586 \mathrm{bp}$ fragment using specific primers for glyceraldehyde 3-phosphate dehydrogenase (GAPDH). PCR products were subjected to electrophoresis on a $1 \%$ agarose gel and stained with ethidium bromide. The intensity of the bands was quantified as arbitrary OD units using the UVP Bioimaging System (Labworks ${ }^{\mathrm{TM}}$, Version 4.6, Upland, CA, USA). The primers were as follows: MMP-2, forward 5'-CTTCATCGC TGCACACCAGG-3' and reverse 5'-GGTATCTGGGCAGC AGAAAG-3'; MMP-9, forward 5'-CGTCTGAGAATTG AATCAGC-3' and reverse 5'-CTAGCACACATGCTGTA TAC-3'; GAPDH, forward 5'-CATGAGGTAGTCTGTCAG GTC-3' and reverse 5'-GATATCGCTGCGCTCGTCGTC-3'.

MMP-9 Western blot analysis. Frozen muscle tissue samples were homogenized in lysis buffer $[0.5 \%$ Nonidet P-40, $10 \mathrm{mM}$ Tris- $\mathrm{HCl}$ ( $\mathrm{pH} 7.5), 150 \mathrm{M} \mathrm{NaCl}, 1 \mathrm{mM}$ phenylmethylsulfonyl fluoride (PMSF), $5 \mathrm{mM}$ aprotinin, $10 \mathrm{mM} \mathrm{KCl,} 2.0 \mu \mathrm{g} / \mathrm{ml}$ aprotinin and $2.0 \mu \mathrm{g} / \mathrm{ml}$ leupeptin] for $40 \mathrm{~min}$ at $4^{\circ} \mathrm{C}$. A total of $50 \mu \mathrm{g}$ of total protein extracts were subjected to $10 \%$ SDS-PAGE, subsequently transferred to nitrocellulose membranes (Bio-Rad Laboratories, Inc., Hercules, CA, USA) and blocked in Tris-buffered saline with Tween-20 (TBS-T) containing $5 \%$ non-fat dry milk for $1 \mathrm{~h}$ at room temperature. Blots were then incubated overnight at $4^{\circ} \mathrm{C}$ with rabbit polyclonal $\mathrm{IgG}$ antibody to MMP-9 (1:100) (Santa Cruz Biotechnology, Inc., Santa Cruz, CA, USA). The antigen-antibody complexes were visualized using goat anti-rabbit IgG antibodies (1:1000) and the enhanced chemiluminescence ECL detection system (BeyoECL Plus, China). 

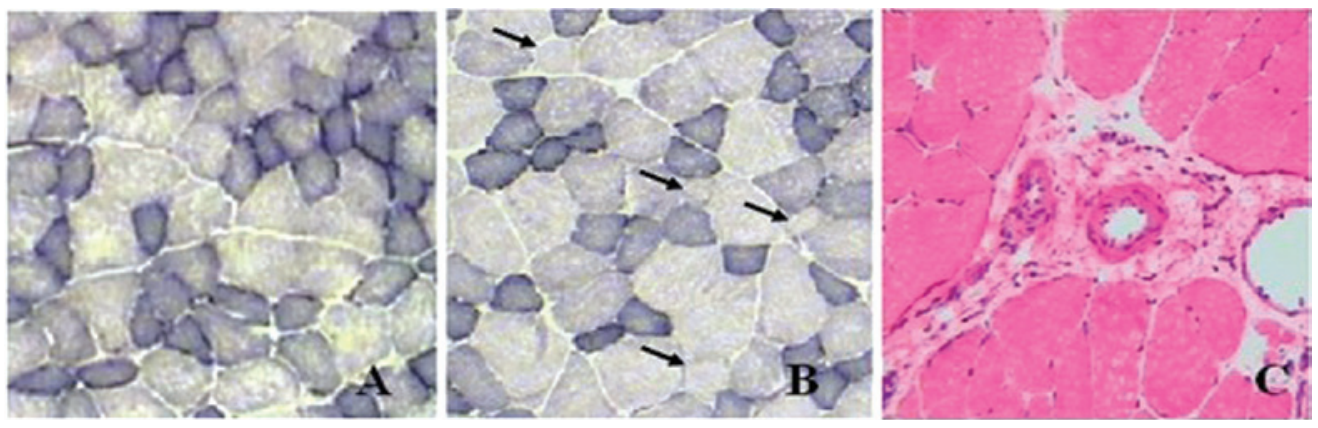

Figure 1. Light micrographs of skeletal muscle sections from rats of the control group and the experimental group. (A) The diameter of the type II fiber of rats is larger than that of type I fibers in the control group (NADH staining, x100). (B) Atrophy of the muscle fibers was observed in the experimental group. The diameter of type II fibers was even smaller than that of type I fibers and the shapes of the fibers became triangular (polygonal) and irregular (NADH staining, x100). (C) Skeletal muscle fibers of the experimental group showed hyperplasia of the connective tissue in the fiber matrix (H\&E staining, x100).
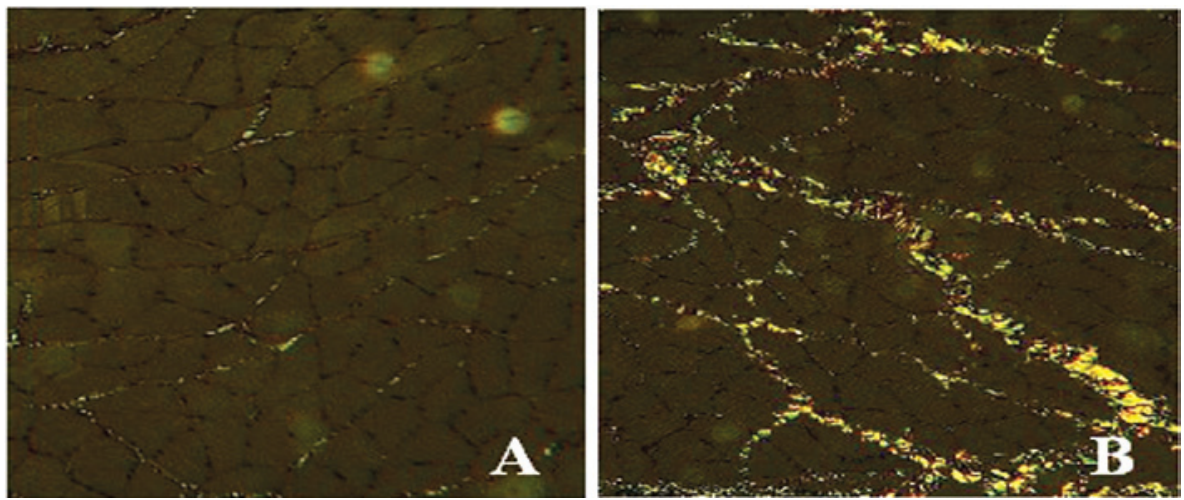

Figure 2. Polarization microscopy of skeletal muscle sections from rats of the control and the experimental groups. (A) There was less intercellular substance between skeletal muscle fibers in the control group (picrosirius red staining, x100). (B) There were hyperplasia of fiber connective tissue in the experimental group (picrosirius red staining, $\mathrm{x} 100$ ).

Zymographic analysis. A total of $20 \mathrm{mg}$ of muscle was washed with cold saline and incubated in $2 \mathrm{ml}$ extraction buffer (10 mM cacodylic acid, pH 5.0, $150 \mathrm{mM} \mathrm{NaCl}, 1 \mathrm{mM} \mathrm{ZnCl}{ }_{2}$, $20 \mathrm{mM} \mathrm{CaCl}_{2}, 1.5 \mathrm{mM} \mathrm{NaN}_{3}$ and $0.01 \%$ Triton X-100) at $4^{\circ} \mathrm{C}$ with continuous mixing for $24 \mathrm{~h}$. Protein concentration was determined by the bicinchoninic acid (BCA) protein assay kit (Beyotime, China), according to manufacturer's instructions. Briefly, equal amounts of total protein (50 $\mu \mathrm{g} /$ lane) were subjected to electrophoresis in triplicate. Zymogram gels consisted of $10 \%$ polyacrylamide impregnated with gelatin at $1 \mathrm{mg} / \mathrm{ml}$. After electrophoresis, the gels were washed 5 times for $50 \mathrm{~min}$ in a $2.5 \%$ Triton $\mathrm{X}-100$ solution and 3 times in Tris buffer $(0.5 \mathrm{~mol} / \mathrm{l}$ Tris-HCl, $\mathrm{pH}$ 7.4) at room temperature. The gels were then incubated for $18 \mathrm{~h}$ in a substrate buffer (50 mM/1 Tris- $\mathrm{HCl}, 20 \mathrm{mM} / 1 \mathrm{NaCl}, 10 \mathrm{mM} / \mathrm{CaCl}_{2}, \mathrm{pH} 7.4$ ) at $37^{\circ} \mathrm{C}$. The gels were then stained in Coomassie Blue R250 for $2 \mathrm{~h}$ and destained in 5\% methanol and $7.5 \%$ acetic acid for $5 \mathrm{~min}$ and then in water overnight. Gelatinolytic activity was identified as clear bands on a blue background. Gelatin zymography detects the activity of both active and pro-form gelatinolytic MMPs. The samples were also assayed in the presence of $15 \mathrm{mM}$ ethylenediaminetetraacetic acid (EDTA) that inhibited MMP activity. The molecular mass of gelatinolytic activities was determined by comparison to reference protein molecular mass marker PageRuler ${ }^{\mathrm{TM}}$ Prestained Protein Ladder (Fermentas Life Sciences, Burlington, ON, Canada). Activity bands were identified following previous descriptions according to their molecular weights $(72 \mathrm{kDa}$, Pro-MMP-2; 64 kDa, active-MMP-2; 92 kDa, Pro-MMP-9; $88 \mathrm{kDa}$, intermediary-MMP-9; $84 \mathrm{kDa}$, active-MMP-9) (11).

Statistical analysis. Standard statistical methods from the SPSS Statistical Analysis System V-9.0 (SPSS, Chicago, IL, USA) were used. Differences between groups were analyzed using ANOVA, the $\chi^{2}$ test and the 2-tailed Student's $t$ test. For all tests, the significance level was set at $5 \%(\mathrm{p}<0.05)$.

\section{Results}

Percentage of success of the model establishment in rats. A total of 4 rats in experimental groups died and the mortality rate was $8.8 \%$ (4/45). Pathological changes of alcoholic myopathy were evident in 29 rats of the experimental group. The percentage of success of the model establishment was 64.4\% (29/45).

Histochemistry studies. Muscle fibers were detected by NADH-TR staining, under which type II fibers appeared light blue and type I fibers appeared dark blue. The shape of the normal muscle fibers of rats is regular and tightly arranged. The diameter of type II fibers is bigger than that of type I fibers (Fig. 1A). Atrophy was evident in the muscle fibers of the experimental group rats, including type I and II fibers. The diameter of type II fibers was even smaller than that of type I fibers and the shapes of the fibers were triangular, polygonal 
Table I. Comparison of the collagen typeI:III ratio in two groups of rats.

\begin{tabular}{|c|c|c|c|c|c|c|c|}
\hline & $\mathrm{N}$ & Collagen area & Collagen IA & MA & Area ratio of I:III & IOD ratio of I:III & CVF (\%) \\
\hline A3 plantaris & 5 & $9085 \pm 1026$ & $483006 \pm 35811$ & $49.16 \pm 8.19$ & $3.14 \pm 1.35$ & $4.35 \pm 1.29$ & $3.68 \pm 1.17$ \\
\hline \multicolumn{8}{|l|}{ B3 } \\
\hline Gastrocnemius & 10 & $13608 \pm 3075^{\mathrm{a}}$ & $699573 \pm 79401^{a}$ & $51.32 \pm 11.43$ & $5.21 \pm 1.38^{\mathrm{a}}$ & $7.08 \pm 1.53^{\mathrm{a}}$ & $6.33 \pm 1.86^{\mathrm{a}}$ \\
\hline Soleus & 10 & $11624 \pm 2132$ & $603541 \pm 36706$ & $51.07 \pm 12.26$ & $5.14 \pm 1.26^{\mathrm{a}}$ & $5.83 \pm 1.36$ & $4.76 \pm 1.59$ \\
\hline Plantaris & 10 & $18006 \pm 4328$ & $1066836 \pm 69024^{b}$ & $59.25 \pm 13.55$ & $8.02 \pm 1.96^{\mathrm{b}}$ & $9.27 \pm 1.83^{b}$ & $8.73 \pm 1.92^{b}$ \\
\hline
\end{tabular}

Compared with control group, $\mathrm{t}=7.96-24.38 ;{ }^{\mathrm{a}} \mathrm{p}<0.05,{ }^{\mathrm{b}} \mathrm{p}<0.01$. A3, control group, untreated skeletal muscle, analyzed 12 weeks later. B3, experimental group, injured skeletal muscle, analyzed 12 weeks later. N, number of rats; MA, medial absorbance; IOD, integral optical density; CVF, collagen volume fraction.

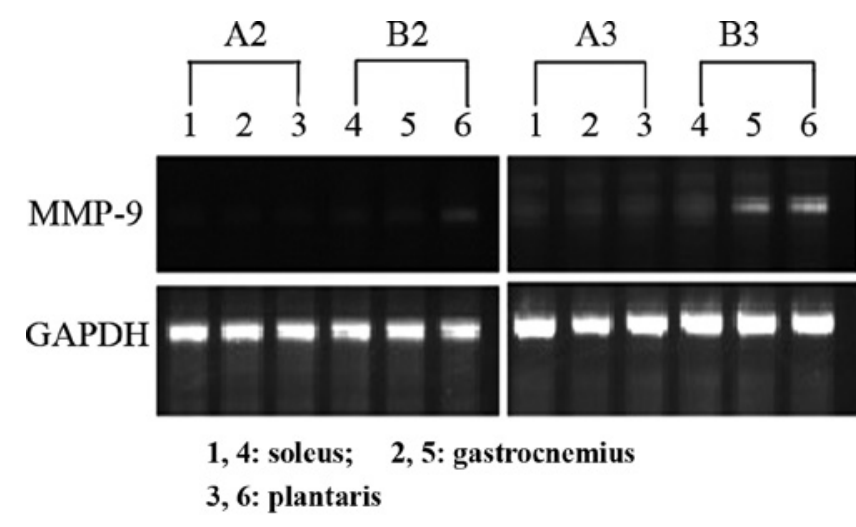

Figure 3. mRNA expression of MMP-9 in prolonged alcoholic myopathy of rats by reverese transcription PCR (RT-PCR)..

and irregular (Fig. 1B). Skeletal muscle fibers of the B groups (gastrocnemius and plantaris) showed hyperplasia of the connective tissue in the fiber matrix (Fig. 1C).

Assessment of picrosirius red-stained sections under polarized light. The relative proportion of skeletal muscle collagen type I and III fibers were compared between group A3 and group B3 by examining picrosirius red-stained sections under polarized light. Fibrotic foci were detected by polarization microscopy. Plantaris fibers of group A3 were arranged compactly (Fig.2A). There was less intercellular substance between plantaris fibers. The collagen type I:III ratio of intercellular substance was normal in group A3. Plantaris fibers of group B3 showed hyperblastosis. There was a significant increase in the levels of fibrosis in group B3 at 12 weeks after administering alcohol (Fig. 2B). For example, the collagen type I:III ratio of intercellular substance was increased in group B3. Importantly, at the later time point, the response to induction of alcohol was significantly different between the experimental group and the control group in the type II skeletal muscle (gastrocnemius and plantaris). These differences included the total collagen area [intergral optic density (IOD)] the collagen type I:III ratio [collagen volume fraction $(\mathrm{CVF})](\mathrm{t}=7.96-24.38, \mathrm{p}<0.05$, Table I). The collagen type I:III ratio was also different in the type I skeletal muscle (soleus) $(\mathrm{p}<0.05$, Table I).

MMP-2 mRNA/MMP-9 mRNA analysis. The mRNA expression of MMP-9 was not observed in the soleus, plantaris and gastrocnemius samples from rats with alcohol exposure at two weeks (data not shown). The mean mRNA expression of MMP-9 (normalized to GAPDH mRNA expression) was greater in the plantaris samples from rats with alcohol exposure at 6 weeks $(0.0579 \pm 0.0046)$, compared with the non-alcohol exposed controls $(0.0198 \pm 0.0023)$. There was a significant difference between the A 2 group and the B2 group ( $\mathrm{t}=-9.151$, $\mathrm{p}=0.001$ ) (Fig. 3). No significant difference was observed between the mRNA expression of MMP-9 in the gastrocnemius and soleus samples from rats with alcohol exposure at 6 weeks compared with the non-alcohol exposed controls (both $\mathrm{p}=0.38$ ). The mean mRNA expression of MMP-9 was greater in the plantaris samples $(0.1417 \pm 0.0116)$ and in the gastrocnemius samples $(0.0599 \pm 0.0057)$ from rats with alcohol exposure at 12 weeks, compared with the non-alcohol exposed controls $(0.0249 \pm 0.0049,0.0237 \pm 0.0021$, respectively) (Fig. 3). There was a significant difference between the A3 group and B3 group ( $\mathrm{t}=-18.095, \mathrm{p}=0.000, \mathrm{t}=-5.933, \mathrm{p}=0.015$, respectively). No significant difference was observed between the mRNA expression of MMP-9 in the soleus samples from rats with alcohol exposure at 12 weeks compared with the non-alcohol exposed controls $(\mathrm{p}=0.8)$.

Western blot analysis. We used Western blotting analysis to determine whether the observed increase in MMP-9 mRNA transcription correlated with MMP-9 protein expression. Analysis of soleus, gastrocnemius and plantaris muscle homogenate from experimental animals demonstrated MMP-9 immunoreactive proteins: a $92 \mathrm{kDa}$ latent form and an active $84 \mathrm{kDa}$ form (Fig. 4). Western blot analysis of muscle extracts with polyclonal antibody against rat MMP-9 revealed the absence of a band at $88 \mathrm{kDa}$ (middle-MMP-9) and $84 \mathrm{kDa}$ (active-MMP-9) and the presence of one band at 92 kDa (pro-MMP-9) at two weeks after injury in all examined muscles. Western blot analysis revealed an upregulation in active MMP-9 in tissue homogenates from the gastrocnemius and plantaris muscle at 6 weeks following alcohol-induced muscle injury compared with the control group. Furthermore, marked upregulation of MMP-9 expression was observed in the gastrocnemius and plantaris at 12 weeks after alcoholinduced muscle injury.

Zymographic analysis. The data of Western blot analysis were supported by MMP-9 gelatin zymogram findings. The 


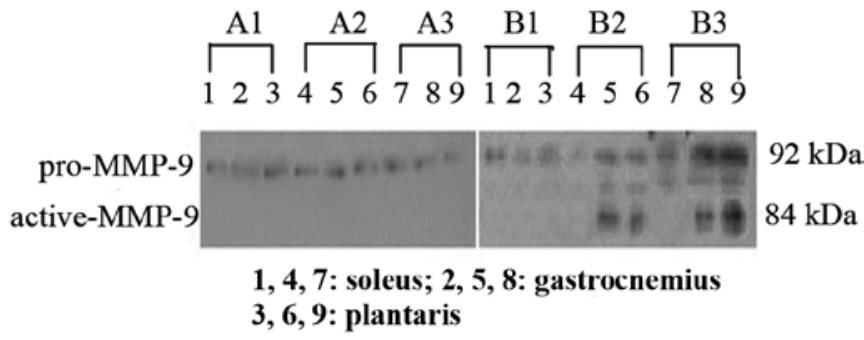

Figure 4. The expression of MMP-9 in prolonged alcoholic myopathy of rats by Western blot analysis.

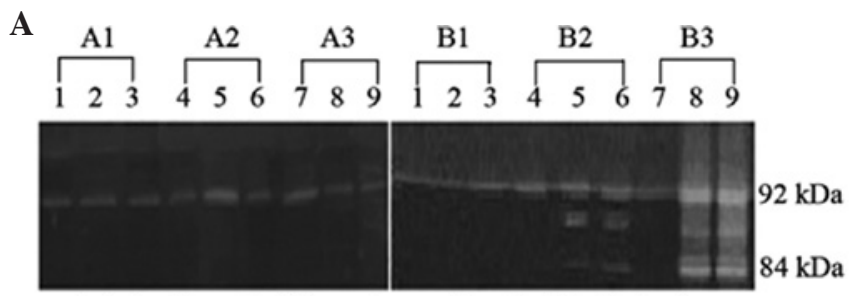

$1,4,7$ : soleus; $2,5,8$ : gastrocnemius; $3,6,9$ : plantaris

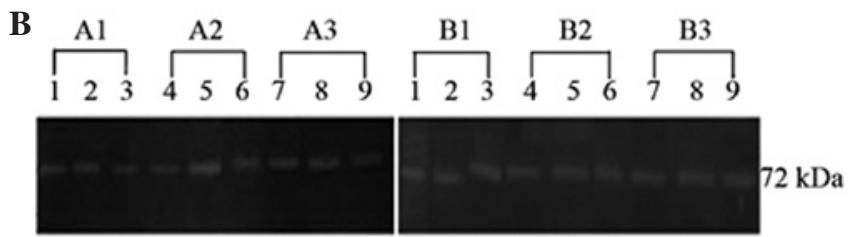

$1,4,7$ : soleus; $2,5,8$ : gastrocnemius; $3,6,9$ : plantaris

Figure 5. Gelatin zymography was used to assess MMP-2 and MMP-9 activity. (A) Bands at $\sim 92 \mathrm{kDa}$ are the pro-form of MMP-9 and the bands just below that are the active form of MMP-9 $(\sim 84 \mathrm{kDa})$. (B) Bands at $72 \mathrm{kDa}$ represent the pro-form of MMP-2.

pro-MMP-9 band was observed in all groups. The MMP-9 activity was elevated in the $\mathrm{B} 2$ group compared to the $\mathrm{A} 2$ group ( $<<0.05$, Fig. 5). In addition, the MMP-9 activity $(84 \mathrm{kDa})$ in the $\mathrm{B} 3$ group was significantly increased when compared with the A3 group ( $p<0.05$, Fig. 5). In particular, gastrocnemius and plantaris from rats showed higher levels of active-MMP-9 12 weeks following alcohol-induced muscle injury compared with the rats at 2 weeks and at 6 weeks after alcohol-induced muscle injury (all $\mathrm{p}<0.05$; Fig. $5 \mathrm{~A}$ ); pro-MMP-2 was observed in all groups. Of note, the MMP-2 activities of the pro-form (72 kDa) from the B1-B3 groups were similar to the A1-A3 groups ( $p>0.05$; Fig. $5 B$ ). No differences were observed between groups $\mathrm{A}$ and $\mathrm{B}$ at the same time points.

\section{Discussion}

The most significant findings of this study are: i) that plantaris and gastrocnemius muscles in the rat subjected to alcohol ingestion show increased expression of MMP-9, but not of MMP-2; ii) that as determined by picrosirius red staining, the increased fibrosis in skeletal muscle is associated with the alcohol exposure in our rat model.

ECM is a three-dimensional network of macromolecules that transmits signals from cells to the ECM and vice versa, mediating cell adhesion, migration, proliferation, differentiation and survival. In the last decade, MMPs have been shown to degrade all ECM components. MMPs, a group of zinc-dependent endopeptidases, are thought to play a central role in the modulation of ECM functions (12). MMPs are commonly induced by cytokine signals as inactive zymogens (pro-forms) that require processing of a prodomain by other MMPs or serine proteinases to attain full activity. Their activities are inhibited by endogenous MMP inhibitors (tissue inhibitors of metalloproteinases; TIMPs-1, $-2,-3$ and -4) (13).

With respect to muscular disorders, particular attention has been paid to a subgroup of MMPs termed gelatinases, comprising MMP-2 (also termed gelatinase A, or $72 \mathrm{kDa}$ type IV collagenase) and MMP-9 (also termed gelatinase B, or $92 \mathrm{kDa}$ type $\mathrm{V}$ collagenase). Earlier reports demonstrated that several MMPs, including MMP-2, 3, 7 and 9, are found in skeletal muscle, possibly playing essential roles in muscle fiber growth and repair by regulating the integrity and composition of ECM in skeletal muscle (3). An increase in MMP production has been associated with skeletal muscle damage. MMP-2 is upregulated in Duchenne muscular dystrophy (DMD) skeletal muscle and is expressed by mesenchymal fibroblastic cells (14). Upregulation of MMP-2 and MMP-9 has also been observed in skeletal muscle of dystrophin-deficient mdx mice (15), an animal model of DMD. Moreover, it has been reported that MMP-2 and MMP-9 are able to process $\beta$-dystroglycan and disrupt the link between the ECM and the cell membrane via the dystroglycan complex in the skeletal muscle from DMD and sarcoglycanopathy patients (16).

In this study, we demonstrated by Western blot analysis and RT-PCR that the skeletal muscles (plantaris and gastrocnemius) contain high amounts of MMP-9 protein and mRNA, but not of MMP-2 protein or mRNA, which is confirmed by the fact that the gelatinolytic bands of MMP-2 and MMP-9 correspond to the molecular weight of the activated (cleaved) MMPs. Importantly, at the same time point, the expression of MMP-9 in the gastrocnemius and plantaris after exposure of rats to alcohol was significantly different between the experimental and the control groups. These results extend previous findings that chronic alcoholic myopathy is characterized by selective atrophy of type II (glycolytic, fast-twitch, anaerobic) fibers (17).

Previous studies have shown that in some inflammatory myopathies, MMP-9 is expressed primarily by invading $\mathrm{T}$ lymphocytes, and is implicated in the pathogenesis of these myopathies (4). MMP-9 activation is related to the early inflammatory phase and to the activation of satellite cells (11). In the present study alcohol caused activation of MMP-9, but not of MMP-2. Differences in the pattern of MMP activation may be related to the severity of skeletal muscle injury. For example, when irreversible injury results from intense expression of cytokines, this may activate MMPs which may be released by infiltrating inflammatory cells, as well as proteases that activate MMPs. We found increases in MMP content and activity following 6 weeks of alcohol exposure. Differences in MMP activation may also be attributed to greater oxidant stress and free radical generation (18). The oxidative stress associated with alcohol is a potentially significant factor in MMP activation. Therefore, we chose the end of the alcohol exposure period (12 weeks of alcohol exposure) as the single time point most likely to demonstrate MMP activation in this experimental protocol. 
Vracko et al showed that MMP-9 caused basement membrane damage upon immunohistological examination (19). Therefore, it is possible that active MMP-9 acted upon matrix components (e.g., elastin and proteoglycans) and resulted in basement membrane damage in rats with alcohol exposure, although this was not specifically examined in this study. In conclusion, we propose that the expression of MMP-9 is implicated in the pathogenesis of prolonged alcoholic myopathy.

\section{Acknowledgements}

This study was supported by grants from the Science and Technology Research Project of Technology Bureau, Liaoning Province, China (No. 20102040) and the Science and Technology Research Project of Technology Bureau, Dalian, Liaoning Province, China (No. 2007E21SF186).

\section{References}

1. Preedy VR, Ohlendieck K, Adachi J, Hunter R, Koll M, Mantle D, Peters TJ, Rajendram R and Sneddon A: The importance of alcohol-induced muscle disease. J Muscle Res Cell Motil 24: 55-63, 2003.

2. Visse R and Nagase H: Matrix metalloproteinases and tissue inhibitors of metalloproteinases: structure, function, and biochemistry. Circ Res 92: 827-839, 2003.

3. Carmeli E, Moas M, Reznick AZ and Coleman R: Matrix metalloproteinases and skeletal muscle. Muscle Nerve 29: 191-197, 2003.

4. Schoser BG, Blottner D and Stuerenburg HJ: Matrix metalloproteinases in inflammatory myopathies: enhanced immunoreactivity near atrophic myofibers. Acta Neurol Scand 105: 309-313, 2002.

5. Schoser BG and Blottner D: Matrix metalloproteinases MMP-2, MMP-7 and MMP-9 in denervated human muscle. Neuroreport 10: 2795-2797, 1999.

6. Sillanaukee P, Kalela A, Seppa K, Hoyhtya M and Nikkari ST: Matrix metalloproteinase- 9 is elevated in serum of alcohol abusers. Eur J Clin Invest 32: 225-229, 2002.
7. Preedy VR and Peters TJ: The effect of chronic ethanol ingestion on protein metabolism in type-I- and type-II-fibre-rich skeletal muscles of the rat. Biochem J 254: 631-639, 1988.

8. Bonner AB, Marway JS, Swann M and Preedy ER: Brain nucleic acid composition and fractional rates of protein synthesis in response to chronic ethanol feeding: comparison with skeletal muscle. Alcohol 13: 581-587, 1996.

9. Lieber CS and DeCarli LM: Animal models of chronic ethanol toxicity. Methods Enzymol 233: 585-594, 1994.

10. Dolber PC and Spach MS: Picrosirius red staining of cardiac muscle following phosphomolybdic acid treatment. Stain Technol 62: 23-26, 1987.

11. Fukushima K, Nakamura A, Ueda H, Yuasa K, Yoshida K, Takeda S and Ikeda S: Activation and localization of matrix metalloproteinase-2 and -9 in the skeletal muscle of the muscular dystrophy dog (CXMDJ). BMC Musculoskelet Disord 8: 54, 2007.

12. Murphy $\mathrm{G}$ and Nagase $\mathrm{H}$ : Progress in matrix metalloproteinase research. Mol Aspects Med 29: 290-308, 2008.

13. Gomis-Ruth FX: Structural aspects of the metzincin clan of metalloendopeptidases. Mol Biotechnol 24: 157-202, 2003.

14. Von Moers A, Zwirner A, Reinhold A, Brueckmann O, van Landeghem F, Stoltenburg-Didinger G, Schuppan D, Herbst $\mathrm{H}$ and Schuelke M: Increased mRNA expression of tissue inhibitors of metalloproteinase-1 and -2 in Duchenne muscular dystrophy. Acta Neuropathol 109: 285-293, 2005.

15. Nakamura A, Yoshida K, Ueda H, Takeda S and Ikeda S: Up-regulation of mitogen activated protein kinases in $\mathrm{mdx}$ skeletal muscle following chronic treadmill exercise. Biochim Biophys Acta 1740: 326-331, 2005.

16. Zhong D, Saito F, Saito Y, Nakamura A,Shimizu T and Matsumura K: Characterization of the protease activity that cleaves the extracellular domain of beta-dystroglycan. Biochem Biophys Res Commun 345: 867-871, 2006.

17. Lang CH, Frost RA, Summer AD and Vary TC: Molecular mechanisms responsible for alcohol-induced myopathy in skeletal muscle and heart. Int J Biochem Cell Biol 37: 2180-2195, 2005.

18. Wang J, Chu H, Zhao H, Cheng X, Liu Y, Jin W, Zhao J, Lin B, Ding Y and Ma H: Nitricoxide synthase-induced oxidative stress in prolonged alcoholic myopathies of rats. Mol Cell Biochem 304: 135-142, 2007.

19. Vracko R, Cunningham D and Frederickson RG: Basal lamina of rat myocardium. Its fate after death of cardiac myocytes. Lab Invest 58: 77-87, 1988 . 